\title{
Production of doubly charmed exotic hadrons in heavy ion collisions
}

\author{
Yuanyuan Hu®, ${ }^{1,2}$ Jinfeng Liao, ${ }^{3, *}$ Enke Wang, ${ }^{1,2, \dagger}$ Qian Wang $\odot,{ }^{1,2, \star}$ Hongxi Xing, ${ }^{1,2, \S}$ and Hui Zhang ${ }^{1,2, \|}$ \\ ${ }^{1}$ Guangdong Provincial Key Laboratory of Nuclear Science, Institute of Quantum Matter, \\ South China Normal University, Guangzhou 510006, China \\ ${ }^{2}$ Guangdong-Hong Kong Joint Laboratory of Quantum Matter, Southern Nuclear Science Computing Center, \\ South China Normal University, Guangzhou 510006, China \\ ${ }^{3}$ Physics Department and Center for Exploration of Energy and Matter, Indiana University, \\ 2401 N Milo B. Sampson Lane, Bloomington, Indiana 47408, USA
}

(Received 26 September 2021; accepted 23 November 2021; published 15 December 2021)

\begin{abstract}
Hadron spectroscopy provides direct physical measurements that shed light on the nonperturbative behavior of quantum chromodynamics (QCD). In particular, various exotic hadrons such as the newly observed $T_{c c}^{+}$by the LHCb collaboration, offer unique insights on the QCD dynamics in hadron structures. In this paper, we demonstrate how heavy ion collisions can serve as a powerful venue for hadron spectroscopy study of doubly charmed exotic hadrons by virtue of the extremely charm-rich environment created in such collisions. The yields of $T_{c c}^{+}$as well as its potential isospin partners are computed within the molecular picture for $\mathrm{Pb}-\mathrm{Pb}$ collisions at center-of-mass energy $2.76 \mathrm{TeV}$. We find about three-order-of-magnitude enhancement in the production of $T_{c c}^{+}$in $\mathrm{Pb}-\mathrm{Pb}$ collisions as compared with the yield in proton-proton collisions, with a moderately smaller enhancement in the yields of the isospin partners $T_{c c}^{\prime 0}$ and $T_{c c}^{\prime++}$. The $T_{c c}^{+}$yield is comparable to that of the $X(3872)$ in the most central collisions while shows a considerably stronger decrease toward peripheral collisions, due to a "threshold" effect of the required double charm quarks for $T_{c c}^{+}$. Final results for their rapidity and transverse momentum $p_{T}$ dependence as well as the elliptic flow coefficient are reported and can be tested by future experimental measurements.
\end{abstract}

DOI: 10.1103/PhysRevD.104.L111502

\section{INTRODUCTION}

Because of the color confinement property of quantum chromodynamics (QCD), the theory of strong interaction, experiments can only directly detect color singlet hadrons, instead of fundamental quarks and gluons. As a result, the properties of hadrons, such as the origin of proton mass and spin, structure functions, hadron spectroscopy, and hadron productions/decays in various processes, are very important for understanding the mystery of nonperturbative dynamics in QCD. The study of hadron spectroscopy historically played crucial roles in the development of the conventional quark model, with the classical example of the $\Omega$ baryon discovery that helped establish the model. Today, extensive efforts on hadron spectroscopy have been actively carried

\footnotetext{
*liaoji@indiana.edu

wangek@scnu.edu.cn

qianwang@m.scnu.edu.cn

\$hxing@m.scnu.edu.cn

"Mr.zhanghui@m.scnu.edu.cn
}

Published by the American Physical Society under the terms of the Creative Commons Attribution 4.0 International license. Further distribution of this work must maintain attribution to the author(s) and the published article's title, journal citation, and DOI. Funded by SCOAP ${ }^{3}$. out by experimental collaborations worldwide such as LHCb, BESIII, BelleII, JLab, CMS, ATLAS, with particular interest in the search of possible exotic hadrons.

Recently, the study of hadrons with two (or more) heavy quarks (or antiquarks) has attracted significant attention. Such states, while expected to exist in both conventional quark model and in the exotic sector, are difficult to create and detect experimentally, due to the apparent absence of any heavy-flavor valence quarks in the beam particles and thus highly suppressed production rate. Nevertheless the available beam energy at the Large Hadron Collider (LHC) and the highly capable detectors have started to offer such opportunity, as shown in the observation of the $\Xi_{c c}^{++}$by $\mathrm{LHCb}$ [1]. Just earlier this month, the LHCb collaboration also reported a $J^{P}=1^{+} T_{c c}^{+}$state with significance over $10 \sigma$ in the prompt $D^{0} D^{0} \pi^{+}$invariant mass distribution in the proton-proton $(p p)$ collisions [2,3], which is the first observation of a doubly charmed tetraquark with quark content $c c \bar{u} \bar{d}$. Its mass is very close to the $D^{0} D^{*+}$ and $D^{+} D^{* 0}$ thresholds with width about $410 \mathrm{keV}$. There are many theoretical studies of the open double heavy tetraquark system in the literature, focusing on key issues such as the formation mechanism (i.e., whether the double heavy tetraquark system is bound or not in either molecular picture or compact tetraquark picture) [4-23], the line shapes 
[24-26], the double heavy exotic spectrum [22,27-37], the decay modes/production mechanism [38-43] and their magnetic dipole moments [44]. Detailed measurements on the $p_{T}$, rapidity, multiplicity and centrality dependence could help unravel the production mechanism and the internal structures of these hadrons [45-48]. The observation of the exotics with two or more heavy quarks also provides a way to shed light on the potential symmetry (such as diquarkantiquark symmetry [49-51]). For reviews of the study of these exotic states, we refer to Refs. [52-61].

The crucial "bottleneck" for the creation of doubly charmed hadrons is the need of at least two charm quarks (which require production of two charm-anti-charm pairs in the initial hard scatterings). In this regard, high energy heavy ion collisions can serve as a powerful venue for the production of doubly charmed exotic hadrons by virtue of the extremely charm-rich environment in such collisions. Indeed, a central heavy ion collision at LHC energies could have many dozens of charm and anti-charm quarks available in a single event $[62,63]$. This unique advantage has been shown for the case of $X(3872)$ production in heavy ion collisions [47,64-73]. It was proposed that the centrality dependence of $X(3872)$ yield could help distinguish a large size hadronic molecular scenario from a compact tetraquark scenario [64]. Given that the $T_{c c}^{+}$production requires at least two $c \bar{c}$ pairs while the $X(3872)$ requires at least one pair, the heavy ion collision should be even more advantageous for producing the $T_{c c}^{+}$. In this paper, we demonstrate this by computing the yields of $T_{c c}^{+}$as well as its potential isospin partners $T_{c c}^{++}, T_{c c}^{++}$and $T_{c c}^{\prime 0}$ in $\mathrm{Pb}-\mathrm{Pb}$ collisions at center-of-mass energy $\sqrt{s}=2.76 \mathrm{TeV}$. The closeness of the $T_{c c}^{+}$to the $D^{0} D^{*+}$ and $D^{+} D^{* 0}$ thresholds not only implies its potential molecular picture, but also indicates large isospin breaking effects in its decay [38], which is similar to the case of the $X(3872)$ [74-76] with the nearby $D^{0} \bar{D}^{* 0}+$ c.c. and $D^{+} D^{*-}+$ c.c. thresholds. Given the above, our calculation is performed within the molecular picture and we use the $X(3872)$ yield to set a benchmark for the $T_{c c}^{+}$. As we shall show below, the yield of the $T_{c c}{ }^{1}$ is enhanced by roughly three-order-of-magnitude as compared with the yield in $p p$ collisions and is comparable to that of the $X(3872)$ in the most central collisions while shows a considerably stronger decrease toward peripheral collisions. Furthermore, we will also present results for the rapidity and transverse momentum $p_{T}$ dependence as well as the elliptic flow coefficient that can be tested by future measurements.

\section{FRAMEWORK}

For this study, we adopt the framework developed in Ref. [64] to generate a total of one million minimum bias

\footnotetext{
${ }^{1}$ When the charged property of $T_{c c}$ is not specified, it includes all the states, i.e., $T_{c c}^{++}, T_{c c}^{\prime+}, T_{c c}^{\prime 0}$ and $T_{c c}^{+}$.
}

events from the default version of AMPT transport model for $\mathrm{Pb}-\mathrm{Pb}$ collisions at $\sqrt{s}=2.76 \mathrm{TeV}$ for simulating the production of both $X(3872)$ and the $T_{c c}$ in these collisions. The charmed mesons $D$ and $D^{*}\left(\bar{D}^{*}\right)$ are collected after the hadronization process and coalesced to the $T_{c c}$ states with the following conditions based on the molecular picture: the relative distance within the region $[5 \mathrm{fm}, 7 \mathrm{fm}]^{2}$ and the invariant mass within the region $\left[2 M_{D}, 2 M_{D^{*}}\right]$. In the $D D^{*}$ molecular picture, there are four possible states:

$$
\begin{aligned}
T_{c c}^{\prime 0}: & D^{0} D^{* 0} \quad I=1, \quad I_{3}=-1, \\
T_{c c}^{\prime++}: & D^{+} D^{*+} I=1, \quad I_{3}=1, \\
T_{c c}^{(\prime)+}: & D^{0 /+} D^{*+/ 0} \quad I=0(1), \quad I_{3}=0 .
\end{aligned}
$$

The first two correspond to the isotriplet states $T_{c c}^{\prime 0}$ and $T_{c c}^{+++}[11,77,78]$ that may potentially be produced. The last two $T_{c c}^{+}$and $T_{c c}^{++}$are isospin triplet

$$
T_{c c}^{++}=-\frac{1}{\sqrt{2}}\left(D^{*+} D^{0}+D^{* 0} D^{+}\right)
$$

and singlet

$$
T_{c c}^{+}=-\frac{1}{\sqrt{2}}\left(D^{*+} D^{0}-D^{* 0} D^{+}\right),
$$

respectively $[24,25]$. As the possible interference between the $D^{0} D^{*+}$ and $D^{+} D^{* 0}$ components is not implemented in the simulation framework, the yields of these two $T_{c c}^{(\prime)+}$ are the same. In what follows, we use $T_{c c}^{(\prime)+}$ to denote these two states. The observed double charm exotic state by $\mathrm{LHCb}[2,3]$ is more likely to be isospin singlet $T_{c c}^{+}$, leaving the other unobserved three states challenging and interesting. As the charmed mesons are formed in the AMPT model based on quark flavor content while lacking spin information, the relative yield ratios between, e.g., $D^{*+}$ versus $D^{+}$or that between $D^{* 0}$ and $D^{0}$ need to be estimated from the thermal model relation

$$
R\left(\frac{A}{B}\right) \equiv \frac{\operatorname{Yield}(A)}{\operatorname{Yield}(B)}=e^{-\left(m_{A}-m_{B}\right) / T_{\text {freezeout }}},
$$

with $m_{A}$ and $m_{B}$ the masses of hadrons $\mathrm{A}$ and $\mathrm{B}$, respectively. Here $T_{\text {freezeout }} \simeq 160 \mathrm{MeV}$ is the freeze-out

\footnotetext{
${ }^{2}$ We note this choice may not be unique and the exotic-state yields depend on the choice. Nevertheless, we have used simulations to check the results by varying the choice to other typical molecular distance ranges such as [ $4 \mathrm{fm}, 6 \mathrm{fm}]$ and $[3 \mathrm{fm}, 5 \mathrm{fm}]$. We find the exotic-state yields stay at the same order of magnitude and more importantly all the relative ratios among the exotic-state yields remain little changed against such change. We also note that this is a simplified mechanism to study the robust features of exotic-state productions and implementing a more sophisticated coalescence procedure will be a future task.
} 
temperature. With the physical masses of $D^{(*)+}$ and $D^{(*) 0}$, we find that the relevant fractions to be $29.3 \%$ versus $70.7 \%$ for $D^{*+}$ versus $D^{+}$and $29.2 \%$ versus $70.8 \%$ for $D^{* 0}$ versus $D^{0}$, respectively. To calibrate potential influence associated with this procedure, we estimate the uncertainty of our results by varying the fractions in the regions $[20 \%, 40 \%]$ and $[80 \%, 60 \%]$ for $D^{+}$and $D^{*+}$, respectively. As a sanity check, we also verified that our model simulation results for the total $D+D^{*}$ yields agree with experimental measurements [79].

\section{RESULTS AND DISCUSSIONS}

In this work we focus on estimating the $T_{c c}^{(\prime)+}$ yield from the coalescence of $D^{0} D^{*+}$ and $D^{+} D^{* 0}$ pairs within the aforementioned framework. As a benchmark for comparison, we also estimate the $X(3872)$ production within the same framework as the average yield from coalescence of the $D^{0} \bar{D}^{0 *}, D^{0 *} \bar{D}^{0}, D^{+} D^{-*}, D^{+*} D^{-}$pairs [64]. Additionally the yields of $T_{c c}^{\prime 0}$ and $T_{c c}^{++}$states are computed from coalescence of the $D^{0} D^{* 0}$ and $D^{+} D^{*+}$ pairs, respectively. With a total of one million minimum bias events from our simulations for $\mathrm{Pb}-\mathrm{Pb}$ collisions at $\sqrt{s}=2.76 \mathrm{TeV}$, the inclusive yields of the $X(3872), T_{c c}^{\prime 0}$, $T_{c c}^{++}$, and $T_{c c}^{(\prime)}$ are found to be around 49000,44000 , 44000 , and 50000, respectively, for $\mathrm{R}\left(\frac{D}{D+D^{*}}\right)=70 \%$. The fact that these four are almost of the same order may appear counter-intuitive at first sight. Given that the $c$ and $\bar{c}$ quarks must be pair produced and thus have the same abundance in each event, a naive counting may suggest that there would be more $c \bar{c}$ pairs than $c c$ pairs and thus more likelihood to form $X(3872)$ than $T_{c c}$. Indeed, assuming on average there are $N$ charm and $N$ anticharm quarks generated in a given event, there would be a total of $N^{2} c \bar{c}$ pairs and $N *(N-1) / 2 c c$ pairs. For $N \gg 1$, roughly it is a factor of 2 difference which is also confirmed from our simulations. However, the formation of either $X(3872)$ or $T_{c c}^{(\prime)+}$ in the molecular picture requires a $D$ with a $D^{*}$ instead of the charm quarks/ antiquarks. This changes the counting: assuming a ratio $\mathrm{R}$ for $\frac{D}{D+D^{*}}$ (and similarly for $\frac{\bar{D}}{\bar{D}+\bar{D}^{*}}$ ), there would be roughly $N R$ of $D$ and $N(1-R)$ of $D^{*}$ as well as $N R$ of $\bar{D}$ and $N(1-R)$ of $\bar{D}^{*}$. So in the end one gets a similar count of $N^{2} R(1-R)$ for both $D D^{*}$ pairs and $D \bar{D}^{*}$ pairs. ${ }^{3}$ This helps explain why the inclusive yields of them are fairly close at large $N$, i.e., the central centrality region discussed below.

To see the fireball volume effect on the $T_{c c}$ production, we plot the centrality dependence of their yields in Fig. 1, where a significant decrease from central to peripheral

\footnotetext{
${ }^{3}$ Notice that the yields of the $X(3872)$ (49000 discussed above) is equal to that of the $T_{c c}^{(\prime)+}$ (50000 discussed above) within their statistic uncertainties.
}

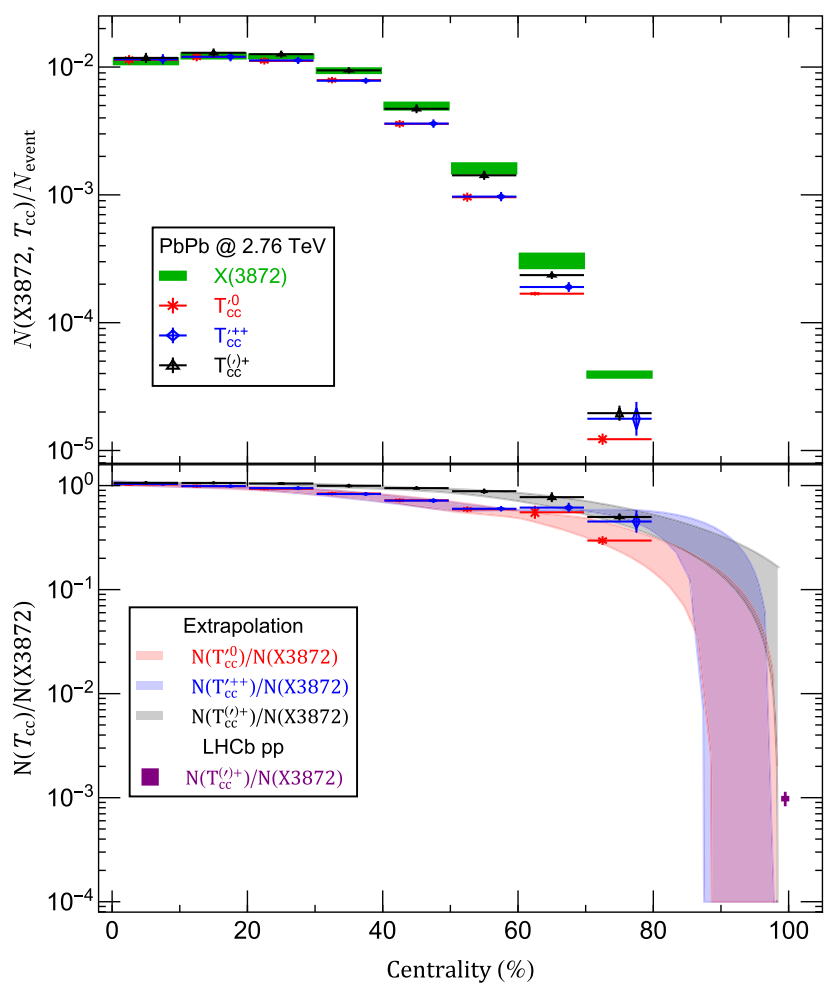

FIG. 1. The centrality dependence of the $X(3872)$ (green solid boxes), $T_{c c}^{\prime 0}$ (red stars), $T_{c c}^{++}$(blue diamonds), and $T_{c c}^{(\prime)+}$ (black triangles) in the $D \bar{D}^{*}+$ c.c. $, D^{0} D^{* 0}, D^{+} D^{*+}$, and $D^{0} D^{*+} / D^{+} D^{* 0}$ hadronic molecular picture, respectively. The bands reflect the uncertainty due to constituent composition as discussed in Eq. (6) that are obtained from varying the composition fraction by $\pm 10 \%$. The ratios of the yields for the $T_{c c}$ s relative to that for the $X(3872)$ are also presented in the lower panel, where an extrapolation with a third-order polynomial function of the $T_{c c}^{\prime 0}$ (gray shaded band) and $T_{c c}^{\prime++}$ (pink shaded band) yield ratios toward ultraperipheral region are also presented. The purple square is the ratio extracted from the experimental data $[2,3,46,81-85]$.

collisions is found. This trend may be expected for a hadron molecule with relatively large size. In heavy ion collisions the charm quarks and antiquarks are carried by bulk flow and the produced charm mesons spread out over the whole fireball [64]. In peripheral collisions the fireball volume becomes small and results in a relatively small spatial separation between the relevant charm mesons, which disfavors the formation of molecular states. Our results for centrality dependence clearly demonstrate the unparalleled advantage of heavy ion collisions for producing the doubly charmed exotic hadrons, especially in the central and semi-central collisions. This might offer exciting opportunity for the search of more such states, e.g., the isospin triplets if they exist.

Furthermore, a comparison between the $T_{c c}$ yields and $X(3872)$ yield as shown by the ratio of the two in Fig. 1 (lower panel) reveals an even stronger suppression of the former in the peripheral collisions. This behavior points to 
an interesting "threshold" effect of the required double charm quarks for $T_{c c}$ formations. Again, let us assume an average of $N$ charm and $N$ anti-charm quarks in a given event with a ratio $\mathrm{R}$ for $\frac{D}{D+D^{*}}$ (and similarly for $\frac{\bar{D}}{\bar{D}+\bar{D}^{*}}$ ). The production of $X(3872)$ requires at least one pair of $D+\bar{D}^{*}$ or $\bar{D}+D^{*}$, for which the probability is $P_{X}=1-R^{2 N}-(1-R)^{2 N}$. The production of $T_{c c}$, on the other hand, requires at least one pair of $D+D^{*}$, for which the probability is $P_{T}=1-R^{N}-(1-R)^{N}$. Note that $R<1$ and $(1-R)<1$, so the chance becomes considerably smaller for $T_{c c}$ production than $X(3872)$ especially when the number $N$ becomes smaller. To given an extreme example: when $N \rightarrow 1$ (i.e., in the limit of only one $c \bar{c}$ pair per event), $P_{T}=0$ while $P_{X}>0$. The essence of such a suppression on the $T_{c c}$ production is essentially a "threshold" effect occurring in the limit of ultra-low charm abundance. In heavy ion collisions, the number $N$ of $c \bar{c}$ pairs per event scales with the number of initial hard scatterings which in turn scales with the so-called binary collision number $N_{\text {coll }}$ [80]. The $N_{\text {coll }}$ drops very rapidly from central toward peripheral region, thus providing an explanation of the observed pattern for $T_{c c}$ centrality dependence.

To gain further insight, we perform an extrapolation of the centrality dependence with a third-order polynomial function for the relative yield ratio between $T_{c c}$ and $X(3872)$ toward the ultraperipheral regime, as indicated by the color bands in Fig. 1 (lower panel). One can see that the yield of the $T_{c c}^{(\prime)+}$ is at least three orders smaller than that of the $X(3872)$ in the ultraperipheral collisions, which are expected to approach the limit of elementary $p p$ collisions. We note that the extrapolated result in that limit shows consistency with the corresponding ratio between $T_{c c}^{(\prime)+}$ and $X(3872)$ in $p p$ collisions from LHCb measurements $[2,3,46,81-85]$. Finally, the extrapolation suggests the yield of the isotriplet states $T_{c c}^{++}$and $T_{c c}^{\prime 0}$ is at least two orders of magnitude smaller than that of the $T_{c c}^{(\prime)+}$, which may provide a plausible reason for the absence of the isotriplet $T_{c c}^{++}$so far in LHCb data [3].

In Fig. 2 we present the rapidity and the transverse momentum distributions of these states, which are found to be similar to those of the usual hadrons $[86,87]$. The rapidity dependence is flat in the middle and decreasing at the forward/backward region. The $p_{T}$ spectra decreases very strongly with increasing $p_{T}$, which may be expected from production from the thermal source with radial flow [64]. Finally we also show the results for the elliptic flow coefficient $v_{2}$ of these states in Fig. 3, which suggest a very similar elliptic flow pattern among these states. The elliptic flow of a particle like $X(3872)$ or $T_{c c}$ would be sensitive to the charm mesons that coalesce into them, especially the spatial distributions of these mesons in the fireball. The similarity in $v_{2}$ among them is due to a similar spatial
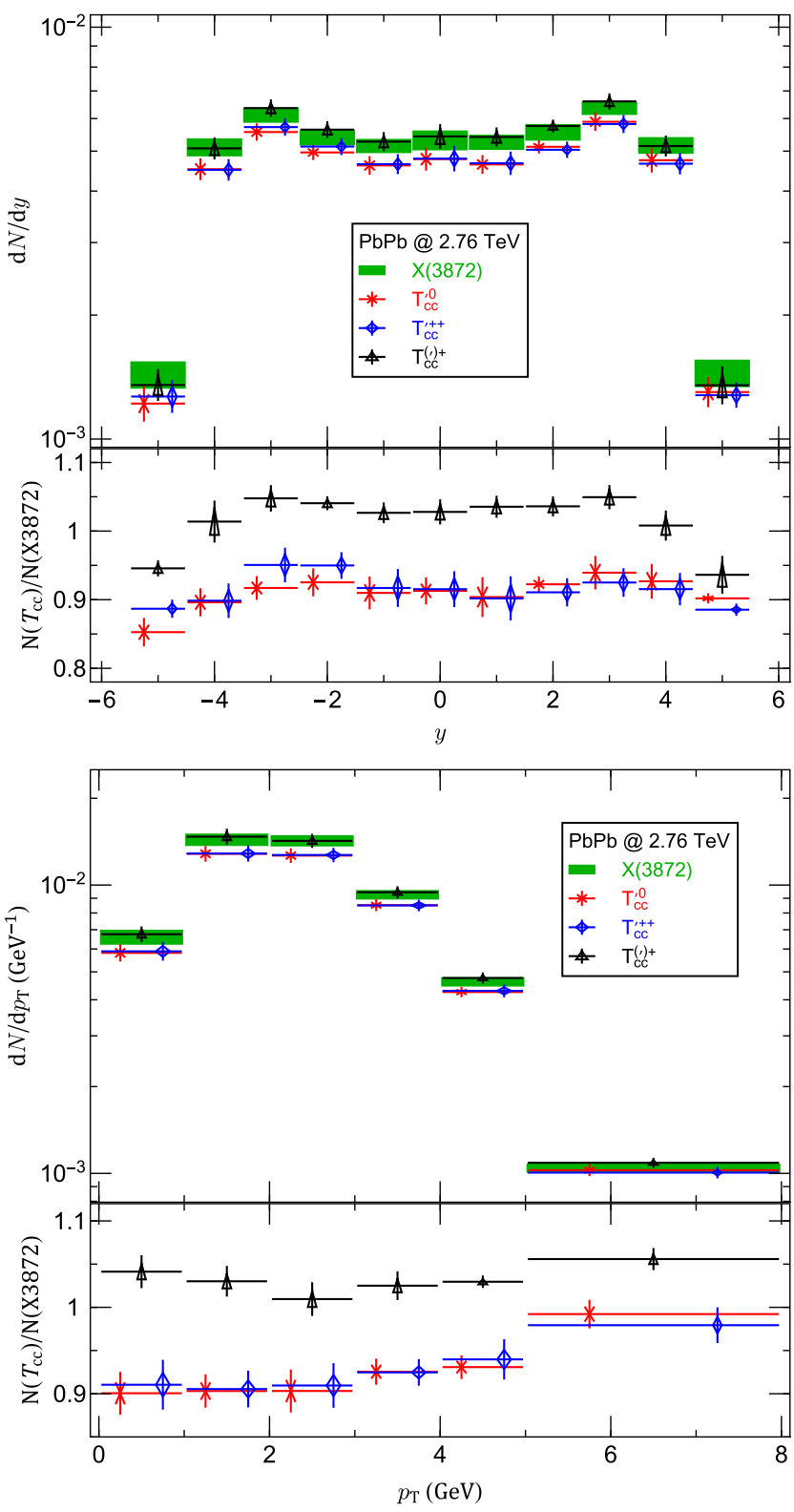

FIG. 2. The rapidity and transverse momentum distributions for the $X(3872)$ (green solid boxes), $T_{c c}^{\prime 0}$ (red stars), $T_{c c}^{\prime++}$ (blue diamonds) and $T_{c c}^{(\prime)+}$ (black triangles) in the $D \bar{D}^{*}+$ c.c., $D^{0} D^{* 0}$, $D^{+} D^{*+}$, and $D^{0} D^{*+} / D^{+} D^{* 0}$ hadronic molecular picture, respectively. The uncertainties are obtained in the same way as that in Fig. 1. The ratios of the yields for the $T_{c c}$ s relative to that for the $X(3872)$ are also presented in the lower panels.

distributions of various $D, D^{*}, \bar{D}$, and $\bar{D}^{*}$ mesons, as we verified from our simulations.

\section{SUMMARY AND OUTLOOK}

In this work, we estimate the yields of the recently observed $T_{c c}^{+}$as well as its potential isospin partners $T_{c c}^{\prime 0}$, $T_{c c}^{\prime+}$ and $T_{c c}^{\prime++}$ within the $D D^{*}$ hadronic molecular picture in $\mathrm{Pb}-\mathrm{Pb}$ collisions at center-of-mass energy $2.76 \mathrm{TeV}$. 


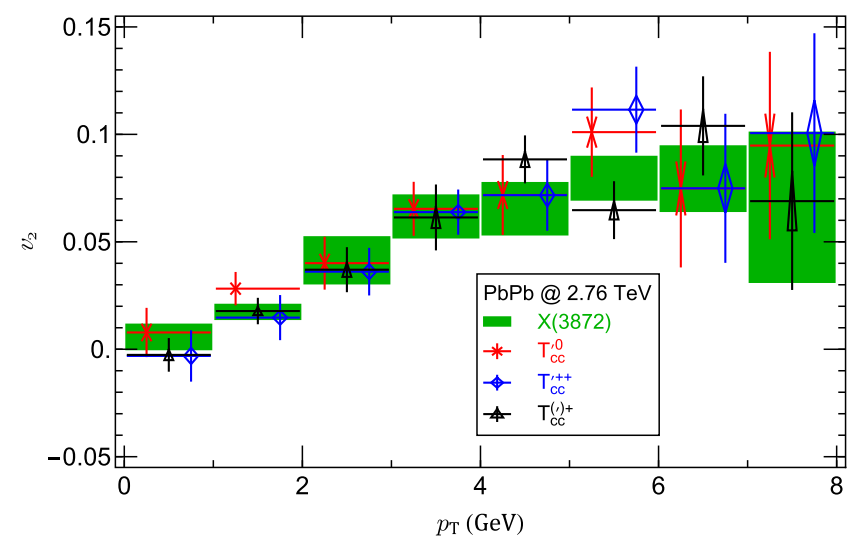

FIG. 3. The elliptic flow coefficient $v_{2}$ versus transverse momentum $p_{T}$ for the $X(3872)$ (green solid boxes), $T_{c c}^{\prime 0}$ (red stars), $T_{c c}^{\prime++}$ (blue diamonds) and $T_{c c}^{(\prime)+}$ (black triangles) in the $D \bar{D}^{*}+$ c.c., $D^{0} D^{* 0}, D^{+} D^{*+}$, and $D^{0} D^{*+} / D^{+} D^{* 0}$ hadronic molecular picture, respectively. The uncertainties are obtained in the same way as that in Fig. 1.

Our main finding is a strong enhancement, about three orders of magnitude, for the $T_{c c}$ yield in the central collisions as compared with the very peripheral collisions which would approach the $p p$ baseline. In comparison with the $X(3872)$ yield computed in the same framework, we find their inclusive yields are close to each other in the central region but the $T_{c c}$ production shows a much stronger suppression into the peripheral region, which can be understood from an interesting "threshold" effect of the required double charm quarks for $T_{c c}$ formation. Final results are obtained for the rapidity and transverse momentum $p_{T}$ dependence of $T_{c c}$ production as well as for the elliptic flow coefficient. Overall, we have demonstrated how heavy ion collisions can serve as a powerful venue for hadron spectroscopy study of doubly charmed exotic hadrons by virtue of the extremely charm-rich environment created in such collisions. It would be exciting to anticipate future experimental efforts that will look for $T_{c c}$ states in heavy ion collisions and test the findings from the present work. Given the advantage of heavy ion collisions in producing an abundance of these doubly charmed exotics, it is conceivable that measurements from heavy ion experiments would offer great opportunities to nail down their structures and properties.

\section{ACKNOWLEDGMENTS}

Discussions with Qingnian $\mathrm{Xu}$ are acknowledged. This work is partly supported by Guangdong Major Project of Basic and Applied Basic Research No. 2020B0301030008, the National Natural Science Foundation of China with Grant No. 12035007, No. 12047523, Science and Technology Program of Guangzhou No. 2019050001, Guangdong Provincial funding with Grant No. 2019QN01X172. Q. W. is also supported by the NSFC and the Deutsche Forschungsgemeinschaft (DFG, German Research Foundation) through the funds provided to the Sino-German Collaborative Research Center TRR110 "Symmetries and the Emergence of Structure in QCD” (NSFC Grant No. 12070131001, DFG Project-ID 196253076-TRR 110). J. L. is supported by the U.S. NSF Grant No. PHY-1913729.
[1] R. Aaij et al. (LHCb Collaboration), Phys. Rev. Lett. 119, 112001 (2017).

[2] R. Aaij et al. (LHCb Collaboration), arXiv:2109.01038.

[3] R. Aaij et al. (LHCb Collaboration), arXiv:2109.01056.

[4] J. P. Ader, J. M. Richard, and P. Taxil, Phys. Rev. D 25, 2370 (1982).

[5] C. Semay and B. Silvestre-Brac, Z. Phys. C 61, 271 (1994).

[6] S. Pepin, F. Stancu, M. Genovese, and J. M. Richard, Phys. Lett. B 393, 119 (1997).

[7] J. Carlson, L. Heller, and J. A. Tjon, Phys. Rev. D 37, 744 (1988).

[8] D. Janc and M. Rosina, Few Body Syst. 35, 175 (2004).

[9] J. Vijande, F. Fernandez, A. Valcarce, and B. Silvestre-Brac, Eur. Phys. J. A 19, 383 (2004).

[10] S. H. Lee and S. Yasui, Eur. Phys. J. C 64, 283 (2009).

[11] Y. Yang, C. Deng, J. Ping, and T. Goldman, Phys. Rev. D 80, 114023 (2009).

[12] F. S. Navarra, M. Nielsen, and S. H. Lee, Phys. Lett. B 649, 166 (2007).

[13] J. Vijande, E. Weissman, A. Valcarce, and N. Barnea, Phys. Rev. D 76, 094027 (2007).
[14] D. Ebert, R. N. Faustov, V. O. Galkin, and W. Lucha, Phys. Rev. D 76, 114015 (2007).

[15] B. A. Gelman and S. Nussinov, Phys. Lett. B 551, 296 (2003).

[16] S. S. Agaev, K. Azizi, and H. Sundu, arXiv:2108.00188.

[17] X. K. Dong, F. K. Guo, and B. S. Zou, Commun. Theor. Phys. 73, 125201 (2021).

[18] Y. Huang, H. Q. Zhu, L.S. Geng, and R. Wang, arXiv:2108.13028 [Phys. Rev. D (to be published)].

[19] N. Li, Z. F. Sun, X. Liu, and S. L. Zhu, Chin. Phys. Lett. 38, 092001 (2021).

[20] H. Ren, F. Wu, and R. Zhu, arXiv:2109.02531.

[21] Z. G. Wang and Z. H. Yan, Eur. Phys. J. C 78, 19 (2018).

[22] Q. Xin and Z. G. Wang, arXiv:2108.12597.

[23] T. Guo, J. Li, J. Zhao, and L. He, arXiv:2108.10462.

[24] M. L. Du, V. Baru, X. K. Dong, A. Filin, F. K. Guo, C. Hanhart, A. Nefediev, J. Nieves, and Q. Wang, arXiv:2110.13765.

[25] V. Baru, X. K. Dong, M. L. Du, A. Filin, F. K. Guo, C. Hanhart, A. Nefediev, J. Nieves, and Q. Wang, arXiv:2110.07484. 
[26] M. Albaladejo, arXiv:2110.02944.

[27] R. J. Hudspith, B. Colquhoun, A. Francis, R. Lewis, and K. Maltman, Phys. Rev. D 102, 114506 (2020).

[28] J. B. Cheng, S. Y. Li, Y. R. Liu, Z. G. Si, and T. Yao, Chin. Phys. C 45, 043102 (2021).

[29] Q. Qin, Y. F. Shen, and F. S. Yu, Chin. Phys. C 45, 103106 (2021).

[30] A. Drutskoy, arXiv:2101.09891.

[31] R. Chen, Q. Huang, X. Liu, and S. L. Zhu, arXiv:2108.01911.

[32] X. Z. Weng, W. Z. Deng, and S. L. Zhu, arXiv:2108.07242.

[33] X. Chen, arXiv:2109.02828.

[34] G. Yang, J. Ping, and J. Segovia, Phys. Rev. D 104, 094035 (2021).

[35] T. W. Wu, Y. W. Pan, M. Z. Liu, S. Q. Luo, X. Liu, and L. S. Geng, arXiv:2108.00923.

[36] K. Chen, R. Chen, L. Meng, B. Wang, and S. L. Zhu, arXiv:2109.13057.

[37] L. R. Dai, R. Molina, and E. Oset, arXiv:2110.15270.

[38] L. Meng, G. J. Wang, B. Wang, and S. L. Zhu, Phys. Rev. D 104, 051502 (2021).

[39] M. J. Yan and M. P. Valderrama, arXiv:2108.04785.

[40] S. Fleming, R. Hodges, and T. Mehen, arXiv:2109.02188 [Phys. Rev. D (to be published)].

[41] Y. Jin, S. Y. Li, Y. R. Liu, Q. Qin, Z. G. Si, and F. S. Yu, Phys. Rev. D 104, 114009 (2021).

[42] X. Z. Ling, M.Z. Liu, L. S. Geng, E. Wang, and J. J. Xie, arXiv:2108.00947.

[43] L. M. Abreu, F. S. Navarra, and H. P. L. Vieira, arXiv:2110 .11145 .

[44] K. Azizi and U. Özdem, Phys. Rev. D 104, 114002 (2021).

[45] J. Crkovska (LHCb Collaboration), Proc. Sci., LHCP2020 (2021) 173.

[46] LHCb Collaboration, Report No. LHCb-CONF-2019-005.

[47] A. Esposito, E. G. Ferreiro, A. Pilloni, A. D. Polosa, and C. A. Salgado, Eur. Phys. J. C 81, 669 (2021).

[48] E. Braaten, L. P. He, K. Ingles, and J. Jiang, Phys. Rev. D 103, L071901 (2021).

[49] M. J. Savage and M. B. Wise, Phys. Lett. B 248, 177 (1990).

[50] N. Brambilla, A. Vairo, and T. Rosch, Phys. Rev. D 72, 034021 (2005).

[51] S. Fleming and T. Mehen, Phys. Rev. D 73, 034502 (2006).

[52] H. X. Chen, W. Chen, X. Liu, and S. L. Zhu, Phys. Rep. 639, 1 (2016).

[53] H. X. Chen, W. Chen, X. Liu, Y. R. Liu, and S. L. Zhu, Rep. Prog. Phys. 80, 076201 (2017).

[54] Y. Dong, A. Faessler, and V. E. Lyubovitskij, Prog. Part. Nucl. Phys. 94, 282 (2017).

[55] R. F. Lebed, R. E. Mitchell, and E. S. Swanson, Prog. Part. Nucl. Phys. 93, 143 (2017).

[56] F. K. Guo, C. Hanhart, U.-G. Meißner, Q. Wang, Q. Zhao, and B. S. Zou, Rev. Mod. Phys. 90, 015004 (2018).

[57] Y. R. Liu, H. X. Chen, W. Chen, X. Liu, and S. L. Zhu, Prog. Part. Nucl. Phys. 107, 237 (2019).

[58] R. M. Albuquerque, J. M. Dias, K. P. Khemchandani, A. M. Torres, F. S. Navarra, M. Nielsen, and C. M. Zanetti, J. Phys. G 46, 093002 (2019).

[59] Y. Yamaguchi, A. Hosaka, S. Takeuchi, and M. Takizawa, J. Phys. G 47, 053001 (2020).
[60] F. K. Guo, X. H. Liu, and S. Sakai, Prog. Part. Nucl. Phys. 112, 103757 (2020).

[61] N. Brambilla, S. Eidelman, C. Hanhart, A. Nefediev, C. P. Shen, C. E. Thomas, A. Vairo, and C. Z. Yuan, Phys. Rep. 873, 1 (2020).

[62] A. Andronic, P. Braun-Munzinger, K. Redlich, and J. Stachel, Phys. Lett. B 571, 36 (2003).

[63] A. Andronic, P. Braun-Munzinger, K. Redlich, and J. Stachel, Nature (London) 561, 321 (2018).

[64] H. Zhang, J. Liao, E. Wang, Q. Wang, and H. Xing, Phys. Rev. Lett. 126, 012301 (2021).

[65] S. Cho et al. (ExHIC Collaboration), Phys. Rev. Lett. 106, 212001 (2011).

[66] B. Chen, L. Jiang, X. H. Liu, Y. Liu, and J. Zhao, arXiv:2107.00969.

[67] A. M. Sirunyan et al. (CMS Collaboration), arXiv:2102 .13048 .

[68] B. Wu, X. Du, M. Sibila, and R. Rapp, Eur. Phys. J. A 57, 122 (2021).

[69] C. E. Fontoura, G. Krein, A. Valcarce, and J. Vijande, Phys. Rev. D 99, 094037 (2019).

[70] J. Hong, S. Cho, T. Song, and S. H. Lee, Phys. Rev. C 98, 014913 (2018).

[71] S. Cho and S. H. Lee, Phys. Rev. C 101, 024902 (2020).

[72] L. M. Abreu and F. J. Llanes-Estrada, Eur. Phys. J. C 81, 430 (2021).

[73] M. Albaladejo, J. M. Nieves, and L. Tolos, Phys. Rev. C 104, 035203 (2021).

[74] D. Gamermann and E. Oset, Phys. Rev. D 80, 014003 (2009).

[75] Z. Y. Zhou and Z. Xiao, Phys. Rev. D 97, 034011 (2018).

[76] N. Li and S. L. Zhu, Phys. Rev. D 86, 074022 (2012).

[77] P. Junnarkar, N. Mathur, and M. Padmanath, Phys. Rev. D 99, 034507 (2019).

[78] R. N. Faustov, V. O. Galkin, and E. M. Savchenko, Universe 7, 94 (2021).

[79] J. Adam et al. (ALICE Collaboration), J. High Energy Phys. 03 (2016) 081.

[80] C. Loizides, J. Kamin, and D. d'Enterria, Phys. Rev. C 97, 054910 (2018); 99, 019901(E) (2019).

[81] R. Aaij et al. (LHCb Collaboration), Eur. Phys. J. C 72, 1972 (2012).

[82] S. Chatrchyan et al. (CMS Collaboration), J. High Energy Phys. 04 (2013) 154.

[83] R. Aaij et al. (LHCb Collaboration), J. High Energy Phys. 08 (2020) 123.

[84] R. Aaij et al. (LHCb Collaboration), Phys. Rev. Lett. 126, 092001 (2021).

[85] B. Heijn, The Measurement of the $\mathrm{X}(3872)$ production cross section via decays to $J / \psi \pi^{+} \pi^{-}$in $p p$ collisions at $\sqrt{s}=13 \mathrm{TeV}$, http://cds.cern.ch/record/2728971.

[86] S. Chatrchyan et al. (CMS Collaboration), J. High Energy Phys. 08 (2011) 141.

[87] E. Abbas et al. (ALICE Collaboration), Phys. Lett. B 726, 610 (2013). 